\title{
Motivating factors and barriers towards exercise in severe mental illness: a systematic review and meta- analysis
}

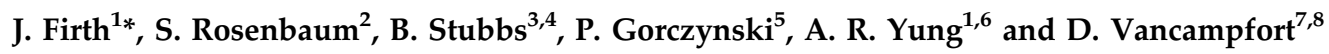 \\ ${ }^{1}$ Institute of Brain, Behaviour and Mental Health, University of Manchester, UK \\ ${ }^{2}$ Department of Exercise Physiology, School of Medical Sciences, Faculty of Medicine, University of New South Wales, Australia \\ ${ }^{3}$ Physiotherapy Department, South London and Maudsley NHS Foundation Trust, UK \\ ${ }^{4}$ Health Service and Population Research Department, Institute of Psychiatry, Psychology and Neuroscience, King's College London, UK \\ ${ }^{5}$ Department of Sport and Exercise Science, University of Portsmouth, UK \\ ${ }^{6}$ Orygen Youth Health Research Centre, University of Melbourne, Australia \\ ${ }^{7} \mathrm{KU}$ Leuven Department of Rehabilitation Sciences, Leuven, Belgium \\ ${ }^{8}$ KU Leuven Department of Neurosciences, UPC KU Leuven, Belgium
}

Exercise can improve clinical outcomes in people with severe mental illness (SMI). However, this population typically engages in low levels of physical activity with poor adherence to exercise interventions. Understanding the motivating factors and barriers towards exercise for people with SMI would help to maximize exercise participation. A search of major electronic databases was conducted from inception until May 2016. Quantitative studies providing proportional data on the motivating factors and/or barriers towards exercise among patients with SMI were eligible. Random-effects meta-analyses were undertaken to calculate proportional data and $95 \%$ confidence intervals (CI) for motivating factors and barriers toward exercise. From 1468 studies, 12 independent studies of 6431 psychiatric patients were eligible for inclusion. Meta-analyses showed that $91 \%$ of people with SMI endorsed 'improving health' as a reason for exercise $(N=6, n=790,95 \%$ CI 80-94). Among specific aspects of health and well-being, the most common motivations were 'losing weight' (83\% of patients), 'improving mood' $(81 \%)$ and 'reducing stress' (78\%). However, low mood and stress were also identified as the most prevalent barriers towards exercise (61\% of patients), followed by 'lack of support' (50\%). Many of the desirable outcomes of exercise for people with SMI, such as mood improvement, stress reduction and increased energy, are inversely related to the barriers of depression, stress and fatigue which frequently restrict their participation in exercise. Providing patients with professional support to identify and achieve their exercise goals may enable them to overcome psychological barriers, and maintain motivation towards regular physical activity.

Received 12 January 2016; Revised 23 June 2016; Accepted 24 June 2016; First published online 9 August 2016

Key words: Exercise, physical activity, physical health, psychosis, schizophrenia.

\section{Introduction}

People with severe mental illness (SMI) experience a premature mortality of around 15-20 years, largely due to inequalities in physical health (Ribe et al. 2014). For instance, people with SMI have a significantly higher risk of obesity, hyperglycaemia and metabolic syndrome, all of which contribute towards the development of cardiovascular diseases (GardnerSood et al. 2015). Many of these physical health issues are related to modifiable risk factors which can be treated and attenuated through lifestyle changes, including exercise and diet (McNamee et al. 2013; Curtis et al. 2016). This is particularly important for those receiving antipsychotic treatment since these

* Address for correspondence: Mr J. Firth, Institute of Brain, Behaviour and Mental Health, University of Manchester, UK.

(Email: joseph.firth@postgrad.manchester.ac.uk) medications greatly increase cardio-metabolic risk when combined with a sedentary lifestyle (McNamee et al. 2013; Vancampfort et al. 2015b).

People with SMI engage in significantly less vigorous exercise, and significantly greater amounts of sedentary behaviour than health controls (Stubbs et al. 2016a, b; Vancampfort et al. 2016a). This inactivity is predictive of a range of adverse health outcomes including obesity, diabetes and medical co-morbidity among people with SMI (Vancampfort et al. 2013a, b; Suetani et al. 2016). It is also associated with more severe negative symptoms and poor socio-occupational functioning (Vancampfort et al. 2012; Suetani et al. 2016).

An increasing body of research demonstrates that exercise interventions can improve physical health and reduce psychiatric symptoms in people with major depression and psychotic disorders (Rosenbaum et al. 2014; Firth et al. 2015). Exercise has also been found

This is an Open Access article, distributed under the terms of the Creative Commons Attribution licence (http://creativecommons.org/licenses/by/4.0/), which permits unrestricted re-use, distribution, and reproduction in any medium, provided the original work is properly cited. 
to reduce negative symptoms and cognitive deficits in schizophrenia (Firth et al. 2015; Kimhy et al. 2015); aspects of the illness which are often left untreated and particularly influential on long-term functioning (Galletly, 2009; Arango et al. 2013). Thus, proper implementation of exercise within the care of people with SMI could reduce cardio-metabolic risk and the associated mortality, while also facilitating functional recovery.

The optimal modality of exercise interventions for people with SMI is yet to be established. A recent meta-analysis suggests that various exercise modalities can be effective for improving outcomes in SMI, although only if a sufficient total volume of activity is achieved (Firth et al. 2015). Clinical trials have also found that significant benefits for depressive and psychotic symptoms only occur among participants who achieve sufficient amounts of exercise (Hoffman et al. 2011; Scheewe et al. 2013). Therefore, training programmes which can maximize adherence to exercise in SMI may be the most effective.

Meta-syntheses of the qualitative literature have previously examined the factors which may encourage or prevent exercise participation among people with SMI (Mason \& Holt, 2012; Soundy et al. 2014a). For instance, improving self-identity and body image is a valued outcome of exercise programmes, while the sedative effects of psychotropic medications can inhibit physical activity (Mason \& Holt, 2012; Soundy et al. 2014a). Although valuable, qualitative investigations can be influenced by interviewers' biases, and results may only represent a subset of the population. Data from survey-based studies may therefore provide a more accurate representation of the entire patient group.

Improving our understanding of desired outcomes of exercise among people with SMI could enhance health promotion initiatives, and inform the development of interventions that are both motivating and rewarding for patients. Furthermore, determining the most common barriers would help to optimize resource allocation when delivering exercise services in clinical practice. Thus, we conducted a systematic review of studies reporting quantitative data on motivating factors and barriers towards exercise for people with SMI. We also quantified patients' responses in these surveys using meta-analytical techniques to determine which were most pertinent for this patient group.

\section{Method}

\section{Search strategy and selection criteria}

An electronic database search of Ovid Medline, Allied and Complementary Medicine Database (AMED),
PsycINFO, EMBASE, and the Health Management Information Consortium (HMIC) database, using the search algorithm: 'exercise' or 'physical activity' or 'sport*' AND 'psychiatric' or 'severe mental' or 'serious mental' or 'schizophrenia' or 'psychosis' or 'bipolar' or 'manic depress" or 'major depress" or 'clinical depress" or 'depressive disorder' AND 'motiv"' or 'barriers' or 'incentives' or 'attitudes' or 'preferences' or 'advantages' or 'disadvantages' was conducted in May 2016, considering articles published from database inception. A search of Google Scholar was conducted using the same key words to identify any additional relevant articles. The reference lists of retrieved articles were also searched.

Only English-language research articles in peerreviewed journals were included in this review. Eligible samples were those in which $>80 \%$ of the sample had a diagnosis of a SMI (i.e. schizophrenia, schizoaffective disorder, other psychotic disorders, bipolar disorder or major depressive disorder) and/or were currently receiving treatment for SMI. Studies which inferred the presence of SMI solely from participants' response to screening questionnaires were excluded if no diagnosis or current treatment for SMI could be confirmed. Eligible studies were those reporting proportional data on motivating factors and/or barriers towards physical activity among people with SMI, from questionnaires, surveys or other quantitative methods. Studies which used only qualitative methods were not eligible for inclusion, as these have been comprehensively reviewed elsewhere (Mason \& Holt 2012; Soundy et al. 2014a). 'Motivating factors' were defined as any outcome of exercise perceived by patients to be a reason for increasing physical activity. 'Barriers' were defined as any physiological, psychological or socio-ecological conditions reported to reduce patients' participation in exercise.

\section{Data extraction and data analysis}

Articles were screened by two reviewers (J.F. and S.R.) to assess eligibility. Disagreements on eligibility were resolved through discussion. A systematic tool was developed (see Supplementary Table S1) to extract all relevant quantitative data from each study into the following categories:

(1) Motivating factors for exercise

(a) Physical: physical health; fitness; strength; weight loss.

(b) Psychological: well-being; enjoyment; reduce distress; mood; self-esteem.

(c) Socio-ecological: socializing; health professional advice; routine.

(2) Barriers to exercise

(a) Physical: physical illness; tiredness/fatigue. 
(b) Psychological: distress; depression; motivational; self-confidence; safety.

(c) Socio-ecological: cost; access to facilities; time; support; insufficient information.

Information on study characteristics (sample size, demographics, location, care setting) was also extracted from each study, and is summarized in Table 1.

\section{Data synthesis and meta-analysis}

We sought to establish the overall prevalence of motivating factors or barriers towards exercise proportion among people with SMI. Therefore, where any specific motivating factor/barrier had been examined by $\geqslant 3$ independent studies, data was pooled using proportional meta-analysis in StatsDirect 2.7 (StatsDirect, 2005). A random-effects model was applied in all meta-analyses, in order to account for expected heterogeneity between studies (DerSimonian \& Laird, 1986). The degree of variance between studies was assessed with Cochran's $Q$ and indexed as $I^{2}$, which estimates the amount of variance caused by between-study heterogeneity, rather than chance. As wording of questions can differ between studies, combinability of study data for meta-analyses was first established through agreed selection by two reviewers (J.F. and S.R.).

\section{Search results}

Fig. 1 shows the full study selection process. The initial database search returned 1534 results. This was reduced to 1163 after duplicates were removed. A further 1109 articles were excluded after reviewing the titles and abstracts for eligibility. Full text versions were retrieved for 54 articles, of which nine were eligible for inclusion. A further three articles were identified from a similar search of Google Scholar. A total of 12 different studies articles, each with unique samples were eligible for inclusion (Faulkner et al. 2007; Ussher, 2007; Sylvia et al. 2009; Gorczynski et al. 2010; Kane et al. 2012; Wynaden et al. 2012; Carpiniello et al. 2013; Bassilios et al. 2014; Deighton \& Addington 2014; Fraser et al. 2015; Klingaman et al. 2014; Firth et al. 2016a). Additional data was obtained for four studies from the corresponding authors (Sylvia et al. 2009; Gorczynski et al. 2010; Deighton \& Addington, 2014; Firth et al. 2016a).

\section{Included studies and participant details}

Characteristics of included studies are detailed in Supplementary Table S2. Three were conducted in the United States, three in Canada, three in Australia, two in the UK, and one in Italy. There were a total of 6431 psychiatric patients within these studies; $85.5 \%$ with schizophrenia, $6.2 \%$ with an unspecified SMI, $2.3 \%$ with bipolar or major depression, and $6 \%$ other/ unknown diagnosis. Where specified, $65 \%$ were community-based outpatients while $35 \%$ were inpatients within psychiatric units. The median age was 42.6 years (range $=19.8-55$ years). Samples ranged from $26-86 \%$ male $($ median $=62 \%$ ). Of 5757 subjects, $50 \%$ belonged to minority groups within their respective countries, while $50 \%$ were white. Five studies $(n=$ 470) also reported employment, showing that $68 \%$ of participants were unemployed. All survey items which were combined for meta-analyses are highlighted in Tables 1 and 2.

\section{Physical health motivations}

Meta-analyses of proportional data are displayed in Fig. 2. The most endorsed reason for exercising was to improve general physical health; endorsed by $91 \%$ of people with SMI $(N=6, n=790,95 \%$ CI 80-94, $Q=$ $\left.81, p<0.01, I^{2}=94 \%\right)$. Two studies which examined motivations for exercise using Likert scales also found that general health improvement ranked higher than all other options (Faulkner et al. 2007; Gorczynski et al. 2010).

Increasing fitness/energy was the most widely assessed physical health motivation $(N=5, n=549)$. This was a motivating factor for $75 \%$ of respondents (95\% CI 64.9-83.4, $Q=19, p<0.01, I^{2}=79 \%$ ) and ranked as 'highly important' in three Likert-scale studies (Faulkner et al. 2007; Sylvia et al. 2009; Gorczynski et al. 2010). 'Improving appearance' and 'losing weight' were examined in only three studies each, but received high rates of endorsement of $77 \%(n=465,95 \%$ CI 64-88, $\left.Q=13.3, p<0.01, I^{2}=85 \%\right)$ and $83 \%$, respectively $(n=169$, 95\% CI 54-99, $Q=30, p<0.01, I^{2}=93 \%$ ). 'Improving strength' averaged $72 \%$ endorsement $(N=3, n=169$, 95\% CI 55-87, $Q=10, p<0.01, I^{2}=81 \%$ ).

\section{Psychological motivations}

As shown in Fig. 2, overall mental health, reducing stress and managing mood were equally popular motivating factors, with $80 \%(N=6, n=788,95 \%$ CI 62-93, $\left.Q=134, p<0.01, I^{2}=96 \%\right), 78 \%(N=4, n=520,95 \% \mathrm{CI}$ 59-92, $\left.Q=50, p<0.01, I^{2}=94 \%\right)$ and $81 \%(N=3, n=$ $464,95 \%$ CI 62-93, $Q=32, p<0.01, I^{2}=94 \%$ ) of patients agreeing, respectively. Improved sleeping patterns was a motivating factor for $72 \%$ of patients $(N=3, n=464$, 95\% CI 55.6-86, $Q=20, p<0.01, I^{2}=90 \%$ ). Enjoyment of exercise was only endorsed by $54 \%$ of respondents ( $n=807,95 \%$ CI 42.5-64.6, $Q=53, p<0.01, I^{2}=89 \%$ ). Likert scales studies also found that mental health benefits and enjoyment of exercise scored moderate-to-high for importance as reasons for exercise. The benefits of exercise for self-confidence were assessed in five studies. 


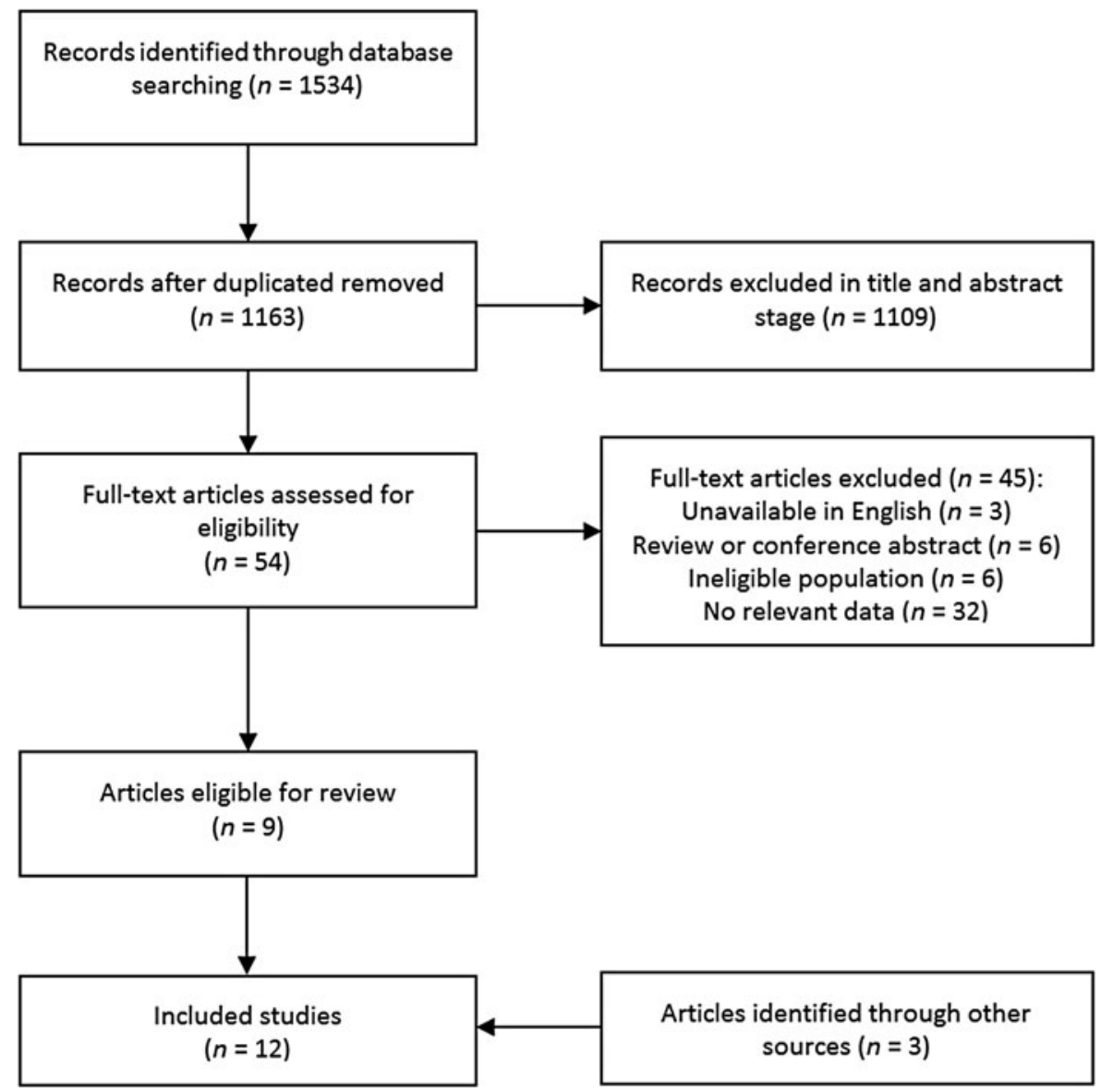

Fig. 1. PRISMA flow diagram of systematic search and study selection.

Although unsuitable for meta-analysis, five studies which assessed the benefits of exercise for self-confidence showed that this is a broadly accepted and valued reason to exercise (See Table 1).

\section{Socio-ecological motivations}

Social aspects of exercise seen as motivating factors by $27 \%$ of patients $(N=3, n=452,95 \%$ CI $23-32, Q=0.1$, $\left.p<0.097, I^{2}=0 \%\right)$. In Likert-scale studies, social aspects scored the lowest of all options presented (Sylvia et al. 2009; Gorczynski et al. 2010; Kane et al. 2012). Similarly, only a minority of participants saw 'improving daily routine' as an important reason for exercise (Sylvia et al. 2009; Wynaden et al. 2012). In contrast, three independent studies found that 'professional support' was perceived as a motivating factor for increasing exercise by the majority of patients (Ussher, 2007; Sylvia et al. 2009; Carpiniello et al. 2013).

\section{Physical health barriers}

Fig. 3 shows meta-analyses of barriers towards exercise. Physical illness and poor health was a barrier for $25 \%$ of participants $(N=3, n=359,95 \%$ CI $10-41$,
$\left.Q=64, p<0.01, I^{2}=92 \%\right)$. Tiredness/low energy was more common, reported by $45 \%$ of patients $(N=5$, $n=6080,95 \%$ CI 25-67, $Q=322, p<0.01, I^{2}=99 \%$ ) and rated as 7.4/10 on relevance scales (Sylvia et al. 2009). Two studies also showed that patients with longterm schizophrenia were more affected by tiredness than healthy controls (Carpiniello et al. 2013; Klingaman et al. 2014). However, this difference did not exist between patients with first-episode psychosis and healthy controls (Deighton \& Addington, 2014).

\section{Psychological barriers}

Proportional meta-analyses showed substantial differences in psychological barriers. 'Stress/depression' was a barrier to exercise for $61 \%$ of respondents $(N=$ 3, $n=5646,95 \%$ CI 43-77, $Q=48, p<0.01, I^{2}=96 \%$ ), whereas 'disinterest in exercise' was a barrier for only $32 \%\left(N=3, n=5822,95 \%\right.$ CI 16-51, $Q=96, p<0.01, I^{2}$ $=98 \%)$. Feeling unsafe and fears of injury were even less common, at $12 \%(N=4, n=5747,95 \%$ CI $9-16, Q$ $\left.=7, p=0.07, I^{2}=57 \%\right)$ and $8 \%(N=3, n=359,95 \% \mathrm{CI}$ 5-11, $\left.Q=0.9, p=0.64, I^{2}=0 \%\right)$, respectively. Data from five studies assessing confidence-related barriers was 
Table 1. Responses to survey items on motivating factors for exercise among people with severe mental illness

\begin{tabular}{|c|c|c|}
\hline Category & Survey item & Average response $^{a}$ \\
\hline \multicolumn{3}{|l|}{ (1) Physical health factors } \\
\hline \multicolumn{3}{|l|}{ General health } \\
\hline Bassilios et al. (2014) & Exercise for physical benefits & $84 \%$ of those intending to exercise \\
\hline Carpiniello et al. (2013) & Exercise is important for physical health & $88 \%$ agreed \\
\hline Deighton \& Addington (2014) & Exercise will make me healthier & $95 \%$ agreed \\
\hline Faulkner et al. (2007) & Improve my health or reduce my risk of disease & Rated $4.3 / 5$ for importance \\
\hline Fraser et al. (2015) & To maintain good health & $98 \%$ agreed \\
\hline Gorczynski et al. (2010) & It would improve my health & Rated $4.3 / 5$ on importance scale \\
\hline Sylvia et al. (2009) & Exercise is beneficial for my physical health & $100 \%$ agreed \\
\hline Ussher (2007) & Exercise is important for physical health & $90 \%$ agreed \\
\hline Wynaden et al. (2012) & 'Why do you attend the gym?' & $61 \%$ said 'to stay healthy' \\
\hline \multicolumn{3}{|l|}{ Fitness/energy } \\
\hline Deighton \& Addington (2014) & I will have more energy & $75 \%$ agreed \\
\hline Faulkner et al. (2007) & It would increase my energy levels & Rated $4.2 / 5$ for importance \\
\hline Firth et al. $(2016 a-c)$ & To increase fitness/energy & $68 \%$ agreed \\
\hline Fraser et al. (2015) & To improve my energy levels & $88 \%$ agreed \\
\hline Gorczynski et al. (2010) & It would help me to stay fit & Rated $4.2 / 5$ on importance scale \\
\hline Gorczynski et al. (2010) & I would have more energy & Rated $3.9 / 5$ on importance scale \\
\hline Kane et al. (2012) & Fitness & Rated $6 / 7$ as a motivating factor \\
\hline Sylvia et al. (2009) & Exercise improves my cardiovascular fitness & $78 \%$ agreed. Avg. rating $=7.7 / 10$ \\
\hline Wynaden et al. (2012) & 'Why do you attend the gym?' (open-answer) & $59 \%$ said 'to get fit' \\
\hline \multicolumn{3}{|l|}{ Strength } \\
\hline Deighton \& Addington (2014) & Exercise makes me feel strong & $83 \%$ agreed \\
\hline Firth et al. $(2016 a-c)$ & To increase sporting ability/strength & $50 \%$ agreed \\
\hline Fraser et al. (2015) & To build up my strength & $81 \%$ agreed \\
\hline \multicolumn{3}{|l|}{ Body weight } \\
\hline Deighton \& Addington (2014) & Exercise will help me lose weight & $80 \%$ agreed \\
\hline Faulkner et al. (2007) & It would help control my weight & Rated $3.8 / 5$ for importance \\
\hline Firth et al. $(2016 a-c)$ & To lose weight & $61 \%$ agreed \\
\hline Fraser et al. (2015) & To control my weight & $98 \%$ agreed \\
\hline \multicolumn{3}{|l|}{ Appearance } \\
\hline Deighton \& Addington (2014) & I will look better & $85 \%$ agreed \\
\hline Faulkner et al. (2007) & It would improve my muscle tone & Rated $4.2 / 5$ for importance \\
\hline Firth et al. $(2016 a-c)$ & To increase muscle tone & $50 \%$ agreed \\
\hline Fraser et al. (2015) & To improve my appearance & $64 \%$ agreed \\
\hline Kane et al. (2012) & Appearance & Rated $5.5 / 7$ as a motivating factor \\
\hline Sylvia et al. (2009) & Exercise improves my body shape and/or tone & $82 \%$ agreed. Avg. rating $=8 \cdot 3 / 10$ \\
\hline \multicolumn{3}{|l|}{ (2) Psychological factors } \\
\hline \multicolumn{3}{|l|}{ General well-being } \\
\hline Bassilios et al. (2014) & Exercise for psychological benefits & $27 \%$ of those intending to exercise \\
\hline Carpiniello et al. (2013) & Exercise is important for mental health & $85 \%$ agreed \\
\hline Deighton \& Addington (2014) & Exercising makes me feel better & $90 \%$ agreed \\
\hline Fraser et al. (2015) & Beneficial for managing psychological well-being & $95 \%$ agreed \\
\hline Fraser et al. (2015) & To give me space to think & $73 \%$ agreed \\
\hline Sylvia et al. (2009) & Exercise is beneficial to my mental health & $99 \%$ agreed \\
\hline Ussher (2007) & Exercise is important for mental health & $72 \%$ agreed \\
\hline Wynaden et al. (2012) & 'Why do you attend the gym?' & $38 \%$ 'to help psychiatric problems' \\
\hline \multicolumn{3}{|l|}{ Enjoyment } \\
\hline Carpiniello et al. (2013) & Enjoys exercise very much so or extremely so & $30 \%$ agreed \\
\hline Deighton \& Addington (2014) & I will have fun & $85 \%$ agreed \\
\hline Firth et al. $(2016 a-c)$ & For having fun & $54 \%$ agreed \\
\hline Fraser et al. (2015) & Because I enjoy exercising & $54 \%$ agreed \\
\hline Gorczynski et al. (2010) & I would have fun & Rated $4 / 5$ on importance scale \\
\hline Kane et al. (2012) & Interest in exercise & Rated $5 / 7$ as a motivating factor \\
\hline Sylvia et al. (2009) & I have fun exercising & $46 \%$ agreed. Avg. rating $=5.6 / 10$ \\
\hline
\end{tabular}


Table 1 (cont.)

\begin{tabular}{|c|c|c|}
\hline Category & Survey item & Average response $^{\mathrm{a}}$ \\
\hline Ussher (2007) & Enjoys exercise very much so or extremely so & $57 \%$ agreed \\
\hline Wynaden et al. (2012) & 'Why do you attend the gym?' & $57 \%$ for 'enjoyment' \\
\hline \multicolumn{3}{|l|}{ Emotions and mood } \\
\hline Deighton \& Addington (2014) & Exercise helps me manage my mood & $75 \%$ agreed \\
\hline Fraser et al. (2015) & To improve my emotional well-being & $94 \%$ agreed \\
\hline Sylvia et al. (2009) & It improves my mood and ability to cope with stress & $70 \%$ agreed. Avg. rating $=7 \cdot 1 / 10$ \\
\hline \multicolumn{3}{|l|}{ Reducing stress } \\
\hline Deighton \& Addington (2014) & Exercise helps me manage stress & $85 \%$ agreed \\
\hline Faulkner et al. (2007) & It would help me feel less tense or stressed & Rated $4.1 / 5$ for importance \\
\hline Faulkner et al. (2007) & It would help me feel less angry or irritable & Rated $3.9 / 5$ for importance \\
\hline Faulkner et al. (2007) & It would take my mind off things & Rated $4 / 5$ for importance \\
\hline Firth et al. $(2016 a-c)$ & Taking your mind off things & $64 \%$ agreed \\
\hline Fraser et al. (2015) & To help manage my stress & $95 \%$ agreed \\
\hline Sylvia et al. (2009) & It improves my mood and ability to cope with stress & $70 \%$ agreed. Avg. rating $=7 \cdot 1 / 10$ \\
\hline Wynaden et al. (2012) & 'Why do you attend the gym?' & $54 \%$ 'to reduce stress' \\
\hline \multicolumn{3}{|l|}{ Self-confidence } \\
\hline Deighton \& Addington (2014) & Exercise makes me feel more self-confident & $88 \%$ agreed \\
\hline Deighton \& Addington (2014) & I will feel better about myself & $90 \%$ agreed \\
\hline Faulkner et al. (2007) & It would improve how I feel about myself & Rated $4.4 / 5$ for importance \\
\hline Firth et al. (2015) & Being more confident in a gym & $64 \%$ agreed \\
\hline Gorczynski et al. (2010) & I would feel better about myself & Rated $3.9 / 5$ on importance scale \\
\hline Sylvia et al. (2009) & Exercise makes me feel good about myself & Rated $7.54 / 10$ for relevance \\
\hline \multicolumn{3}{|l|}{ Sleep } \\
\hline Deighton \& Addington (2014) & I will sleep better & $80 \%$ agreed \\
\hline Faulkner et al. (2007) & It would help me sleep better & Rated $3.9 / 5$ for importance \\
\hline Fraser et al. (2015) & It helps me sleep better & $79 \%$ agreed \\
\hline Sylvia et al. (2009) & I can sleep better if I exercise & $59 \%$ agreed. Avg. rating $=6.6 / 10$ \\
\hline \multicolumn{3}{|l|}{ (3) Socio-ecological factors } \\
\hline \multicolumn{3}{|l|}{ Social aspects } \\
\hline Firth et al. $(2016 a-c)$ & Meeting new people' & $29 \%$ agreed \\
\hline Fraser et al. (2015) & I enjoy the social aspects & $27 \%$ agreed \\
\hline Gorczynski et al. (2010) & People important to me would be happy if I did & Rated $3.7 / 5$ on importance scale \\
\hline Kane et al. (2012) & Social & Rated $3.4 / 7$ as a motivating factor \\
\hline Sylvia et al. (2009) & Exercising is a chance for me to see people & $27 \%$ agreed. Avg. rating $=4.1 / 10$ \\
\hline \multicolumn{3}{|l|}{ Professional support } \\
\hline Carpiniello et al. (2013) & Would exercise more with doctors' advice & $63 \%$ agreed \\
\hline Carpiniello et al. (2013) & Instructor's help would increase levels of exercise & $68 \%$ agreed \\
\hline Fraser et al. (2015) & I exercise because my doctor advised me to & $61 \%$ agreed \\
\hline Ussher (2007) & Would exercise more with doctors' advice & $58 \%$ agreed \\
\hline Ussher (2007) & Instructor's help would increase levels of exercise & $58 \%$ agreed \\
\hline \multicolumn{3}{|l|}{ Daily routine } \\
\hline Sylvia et al. (2009) & I have nothing better to do with my time & Rated $2.87 / 10$ for relevance \\
\hline Sylvia et al. (2009) & Exercise helps to structure my day & Rated $4.96 / 10$ for relevance \\
\hline Wynaden et al. (2012) & 'Why do you attend the gym?' & $45 \%$ 'to get into a routine' \\
\hline Wynaden et al. (2012) & 'Why do you attend the gym?' & $42 \%$ 'to pass time' \\
\hline
\end{tabular}

\footnotetext{
${ }^{a}$ Bold indicates inclusion in meta-analyses.
}

unsuitable for meta-analyses, but collectively showed that this was only a concern for a minority of participants (7-36\%), and to a limited extent; consistently scoring $<2 / 5$ on Likert scales of importance (Table 2).

Data on 'low motivation' was also unsuitable for proportional meta-analysis. However, all three studies which assessed this found that motivational deficits were among the most common psychological barriers towards exercise (Carpiniello et al. 2013; Deighton \& Addington, 2014; Fraser et al. 2015). Furthermore, patients with long-term schizophrenia experienced motivational barriers significantly more than healthy 
Table 2. Responses to items on barriers towards exercise among people with SMI

\begin{tabular}{|c|c|c|}
\hline Category & Survey item & Average response $^{\mathrm{a}}$ \\
\hline \multicolumn{3}{|l|}{ (1) Physical barriers } \\
\hline \multicolumn{3}{|l|}{ Poor physical health } \\
\hline Bassilios et al. (2014) & Physical health problems as a barrier & $28 \%$ of non-vigorous exercisers \\
\hline Carpiniello et al. (2013) & Illness & $19.6 \%$ agreed \\
\hline Fraser et al. (2015) & Physical health problems & $44 \%$ agreed \\
\hline Sylvia et al. (2009) & Exercise will not change my physical health & Avg. rating $=1.7 / 10$ \\
\hline Ussher (2007) & Illness & $15 \%$ agreed \\
\hline Fraser et al. (2015) & Feel too unwell & $60 \%$ agreed \\
\hline \multicolumn{3}{|l|}{ Tiredness/low energy } \\
\hline Carpiniello et al. (2013) & Too tired & $38.4 \%$ agreed \\
\hline Deighton \& Addington (2014) & Lack of energy & 'Sometimes a barrier' \\
\hline Faulkner et al. (2007) & It would leave me feeling tired & Rated $2.1 / 5$ for importance \\
\hline Fraser et al. (2015) & Feel too tired & $74 \%$ agreed \\
\hline Fraser et al. (2015) & Lack of energy & $76 \%$ agreed \\
\hline Klingaman et al. (2014) & Too tired & $27.8 \%$ agreed \\
\hline Sylvia et al. (2009) & I do not have enough energy & $69 \%$ agreed. Avg. rating $=7.4 / 10$ \\
\hline Ussher (2007) & Too tired & $20 \%$ agreed \\
\hline \multicolumn{3}{|l|}{ (2) Psychological barriers } \\
\hline \multicolumn{3}{|l|}{ Stress/depression } \\
\hline Carpiniello et al. (2013) & Unconfident about ability to exercise if sad/stressed & $76.4 \%$ agreed \\
\hline Klingaman et al. (2014) & Stress/depression & $48 \%$ agreed \\
\hline Ussher (2007) & Unconfident about ability to exercise if sad/stressed & $58 \%$ agreed \\
\hline \multicolumn{3}{|l|}{ Low motivation } \\
\hline Carpiniello et al. (2013) & Poor desire & $25.4 \%$ agreed \\
\hline Deighton \& Addington (2014) & Lack of motivation & 'Sometimes a barrier' \\
\hline Fraser et al. (2015) & Lack of motivation & $73 \%$ agreed \\
\hline \multicolumn{3}{|l|}{ Disinterest } \\
\hline Bassilios et al. (2014) & Disinterest as a barrier & $55 \%$ of non-vigorous exercisers \\
\hline Deighton \& Addington (2014) & Lack of programmes that interest me & 'Never or sometimes a barrier' \\
\hline Fraser et al. (2015) & Do not enjoy physical activity & $27 \%$ agreed \\
\hline Klingaman et al. (2014) & Do not like exercise & $22.4 \%$ agreed \\
\hline Sylvia et al. (2009) & I do not have enough interest in exercising & $48 \%$ agreed. Avg. rating $=5.5 / 10$ \\
\hline \multicolumn{3}{|l|}{ Self-confidence } \\
\hline Deighton \& Addington (2014) & Don't like how my body looks & 'Never or sometimes a barrier' \\
\hline Deighton \& Addington (2014) & Failure to achieve exercise goals in the past & 'Never or sometimes a barrier' \\
\hline Deighton \& Addington (2014) & Lack of skills or ability to do a certain type of exercise & 'Never or sometimes a barrier' \\
\hline Faulkner et al. (2007) & I would worry about what other people think of me & Rated $1.4 / 5$ for importance \\
\hline Faulkner et al. (2007) & I would be worried that I would not be very good at it & Rated $2 / 5$ for importance \\
\hline Fraser et al. (2015) & Feel too shy/embarrassed & $36 \%$ agreed \\
\hline Fraser et al. (2015) & Not the sporty type & $29 \%$ agreed \\
\hline Gorczynski et al. (2010) & I feel embarrassed if people see me doing it & Rated $1.7 / 5$ on importance scale \\
\hline Ussher (2007) & Self-consciousness & $7 \%$ agreed \\
\hline \multicolumn{3}{|l|}{ Feeling unsafe } \\
\hline Carpiniello et al. (2013) & Feel unsafe going outdoors & $9 \%$ agreed \\
\hline Deighton \& Addington (2014) & Feeling uncomfortable or intimidated & 'Never or sometimes a barrier' \\
\hline Deighton \& Addington (2014) & Fear of making an existing condition worse & 'Never or sometimes a barrier' \\
\hline Fraser et al. (2015) & Feels unsafe to go outside & $16 \%$ agreed \\
\hline Klingaman et al. (2014) & Safety concerns & $14 \%$ agreed \\
\hline Ussher (2007) & Feel unsafe going outdoors & $9 \%$ agreed \\
\hline \multicolumn{3}{|l|}{ Fear of injury } \\
\hline Carpiniello et al. (2013) & Afraid of getting injured & $5.8 \%$ agreed \\
\hline Deighton \& Addington (2014) & Fear of injury or re-injury & 'Never or sometimes a barrier' \\
\hline Faulkner et al. (2007) & I might injure myself & Rated $2 / 5$ for importance \\
\hline Fraser et al. (2015) & Worried I might get injured & $9 \%$ agreed \\
\hline Ussher (2007) & Afraid of getting injured & $8 \%$ agreed \\
\hline
\end{tabular}


Table 2 (cont.)

\begin{tabular}{|c|c|c|}
\hline Category & Survey item & Average response $^{a}$ \\
\hline \multicolumn{3}{|l|}{ (3) Socio-ecological barriers } \\
\hline \multicolumn{3}{|l|}{ Lack of time } \\
\hline Carpiniello et al. (2013) & Takes too much time & $23.9 \%$ agreed \\
\hline Deighton \& Addington (2014) & Lack of time & 'Never or sometimes a barrier' \\
\hline Faulkner et al. (2007) & It would take time away from other things & Rated 2.1/5 for importance \\
\hline Fraser et al. (2015) & I do not have enough time & $13 \%$ agreed \\
\hline Gorczynski et al. (2010) & It takes time away from doing other things & Rated $2.7 / 5$ on importance scale \\
\hline Klingaman et al. (2014) & Too little time & $13.9 \%$ agreed \\
\hline Klingaman et al. (2014) & Job/work & $5.6 \%$ agreed \\
\hline Klingaman et al. (2014) & Daily routine do not include exercise & $26.5 \%$ agreed \\
\hline Sylvia et al. (2009) & I do not have enough time to exercise & $32 \%$ agreed \\
\hline Ussher (2007) & Takes too much time & $12 \%$ agreed \\
\hline \multicolumn{3}{|l|}{ Lack of support } \\
\hline Carpiniello et al. (2013) & Would receive little help with exercise from others & $65.3 \%$ agreed \\
\hline Deighton \& Addington (2014) & Lack of support from others & 'Never or sometimes a barrier' \\
\hline Gorczynski et al. (2010) & I would need too much help from others & Rated $2.5 / 5$ on importance scale \\
\hline Klingaman et al. (2014) & Lack of support/encouragement & $19.8 \%$ agreed \\
\hline Ussher (2007) & Would receive little help with exercise from others & $68 \%$ agreed \\
\hline \multicolumn{3}{|l|}{ Lack of information } \\
\hline Carpiniello et al. (2013) & Not sure what to do & $15 \%$ agreed \\
\hline Deighton \& Addington (2014) & Lack of knowledge about how to exercise & 'Never or sometimes a barrier' \\
\hline Faulkner et al. (2007) & I don't know how to do physical activities & Rated $1.8 / 5$ for importance \\
\hline Faulkner et al. (2007) & Difficult to find out what to do and where to do it & Rated $2.1 / 5$ for importance \\
\hline Gorczynski et al. (2010) & There is too much I have to learn to do it & Rated $2.3 / 5$ on importance scale \\
\hline Sylvia et al. (2009) & Not know how to exercise/what to do in a gym & $11 \%$ agreed. Avg. rating $=2.9 / 10$ \\
\hline Ussher (2007) & Not sure what to do & $6 \%$ agreed \\
\hline \multicolumn{3}{|l|}{ Cost } \\
\hline Deighton \& Addington (2014) & Cost of physical activity programme & 'Sometimes a barrier' \\
\hline Faulkner et al. (2007) & It would cost too much & Rated $2.4 / 5$ for importance \\
\hline Fraser et al. (2015) & Cost & $19 \%$ agreed \\
\hline Klingaman et al. (2014) & Too little money & $24.7 \%$ agreed \\
\hline \multicolumn{3}{|l|}{ Access to facilities } \\
\hline Deighton \& Addington (2014) & Lack of transportation & 'Never or sometimes a barrier' \\
\hline Deighton \& Addington (2014) & Lack of facilities near by & 'Never or sometimes a barrier' \\
\hline Fraser et al. (2015) & Lack of access to facilities & $41 \%$ agreed \\
\hline Klingaman et al. (2014) & No place to walk or be active & $11.2 \%$ agreed \\
\hline Klingaman et al. (2014) & No transport & $11.8 \%$ agreed \\
\hline \multicolumn{3}{|l|}{ Training partner } \\
\hline Deighton \& Addington (2014) & Do not have anyone to go with & 'Never or sometimes a barrier' \\
\hline Faulkner et al. (2007) & I would have to do it by myself & Rated $2.7 / 5$ for importance \\
\hline Gorczynski et al. (2010) & I would have to do it by myself & Rated $2.8 / 5$ on importance scale \\
\hline Sylvia et al. (2009) & I have no one to exercise with & Rated 2.6/10 for relevance \\
\hline
\end{tabular}

${ }^{a}$ Bold indicates inclusion in meta-analysis.

controls (Carpiniello et al. 2013). Again, however, there was no significant difference in the early stages of illness (Deighton \& Addington, 2014).

\section{Socio-ecological barriers}

The most frequently experienced practical barrier was a 'lack of support', reported by $50 \%$ of respondents
$(N=3, n=5646, \quad 95 \% \quad$ CI 15-86, $Q=240, \quad p<0.01$, $\left.I^{2}=99 \%\right)$. This was significantly more prevalent among schizophrenia patients than healthy controls (Carpiniello et al. 2013; Klingaman et al. 2014). People with first-episode psychosis also scored these items higher than controls, although differences were not statistically significant (Deighton \& Addington, 2014). 'Lack of training partner' was a moderately ranked 


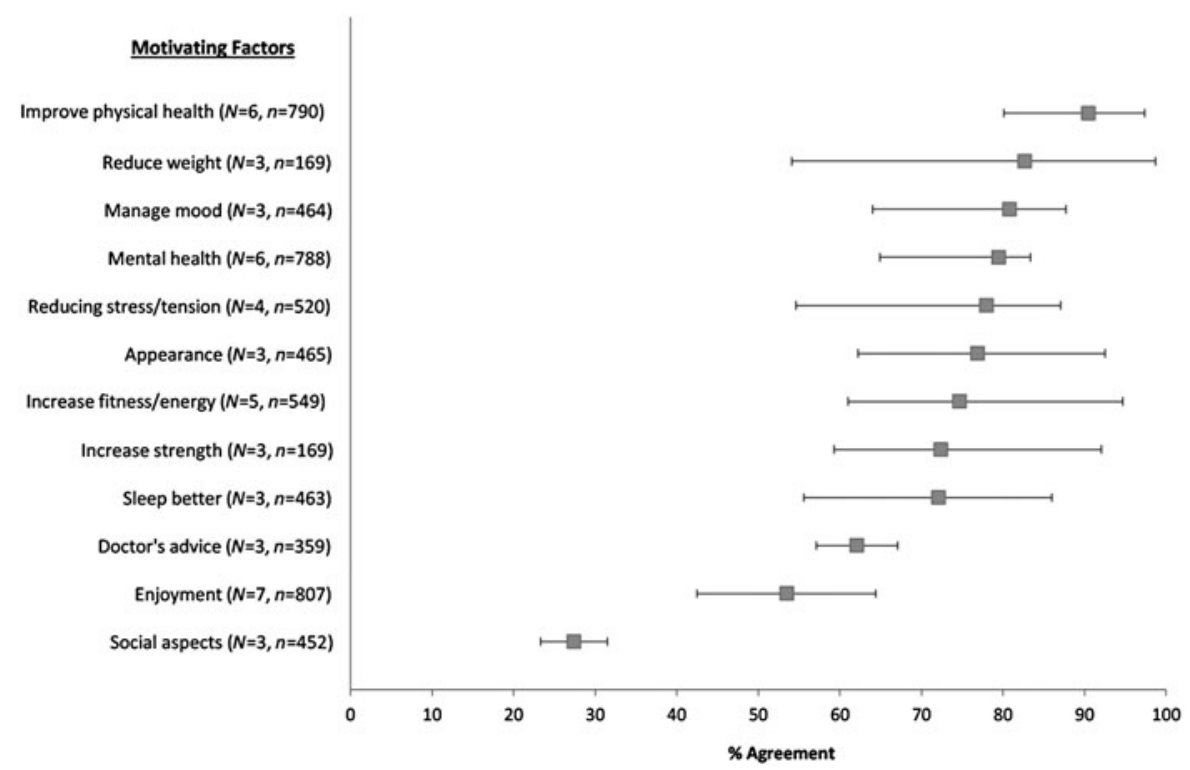

Fig. 2. Proportional meta-analyses of motivating factors for exercise in severe mental illness. The forest plot shows the \% of patients agreeing with each motivating factors (box points) and the $95 \%$ confidence intervals (horizontal lines). Individual study items used in meta-analyses are shown in Table 1.

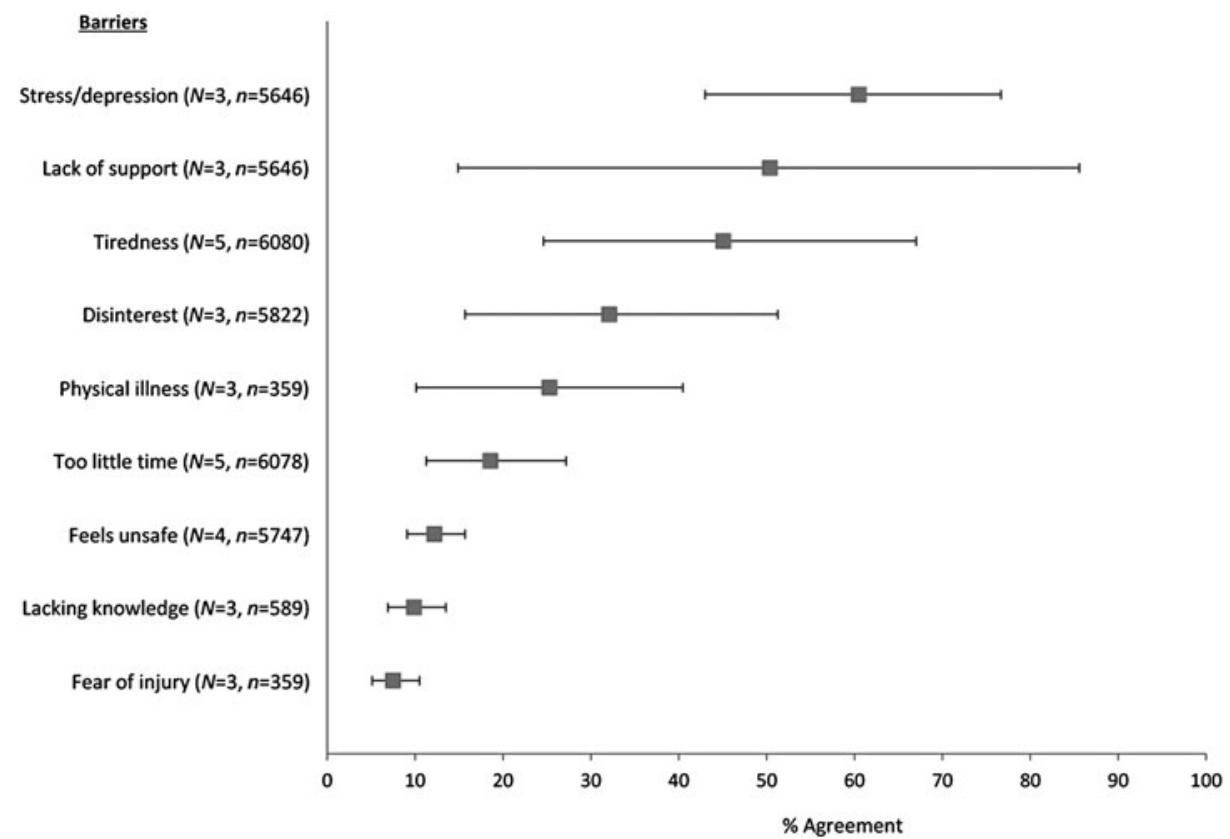

Fig. 3. Proportional meta-analyses of barriers to exercise in severe mental illness. The forest plot shows the \% of patients experiencing each barrier (box points) and the 95\% confidence intervals (horizontal lines). Individual items combined for meta-analysis are shown in Table 2.

barrier, but was regarded as significantly more important by those patients who were interested in increasing their exercise (Faulkner et al. 2007).

'Lack of time' was the most widely investigated practical barrier, although only $19 \%$ of respondents identified this as a barrier $(N=5, n=6078,95 \% \mathrm{CI}$ 11.3-27.2, $\left.Q=68, p<0.01, I^{2}=94 \%\right)$. Three studies using
Likert scales also found that time-related barriers were mostly unimportant (Faulkner et al. 2007; Gorczynski et al. 2010; Deighton \& Addington, 2014). Furthermore, 'lack of time' was significantly less of a barrier for people with SMI than for healthy controls (Deighton \& Addington, 2014; Klingaman et al. 2014). Only 10\% of patients felt that 'lack of exercise information' was a 
barrier ( $n=589,95 \%$ CI 7-14, $Q=3.4, p=0.18, I^{2}=42 \%$ ). Additional data (unsuitable for meta-analysis) on cost and accessibility of exercise services indicated these were of low importance (See Table 2).

\section{Discussion}

The purpose of this study was to examine the motivating factors and barriers towards exercise among people with SMI, in order to inform the design and delivery of interventions aiming to increase exercise participation. A total of 12 studies (of 6431 psychiatric patients with predominantly schizophrenia/schizoaffective disorders) were identified. As nine of the 12 studies reviewed had been conducted from 2013 onwards, the evidence/data presented can be considered timely and up-to-date.

Our results show that the primary incentive for engaging in exercise was to improve physical health (Fig. 2). Specifically, weight loss was the single most popular reason for participating in exercise, comparable to the motivating factors identified by the general population (Sherwood \& Jeffery, 2000), and unsurprising given the high rates of overweight and obesity among people with SMI (Vancampfort et al. 2015b). Although weight management can be a key motivating factor for initiating an exercise programme, it is important to note $(a)$ the relatively modest contribution of physical activity to weight loss beyond that achieved through dietary interventions (Haskell et al. 2007), and (b) that improvements in mental and physical health outcomes in response to exercise interventions are often achieved independent of weight loss (Firth et al. 2015). While weight management may be an important motivating factor for people with SMI to commence an exercise programme, education and support should be provided to ensure long-term adoption and maintenance regardless of any change in body weight achieved. Furthermore, if weight loss is a primary aim, dietary interventions must be provided as part of best-practice lifestyle interventions (Ward et al. 2015).

The high endorsement of 'fitness' as an incentive is encouraging, since this is readily improved by exercise interventions in SMI (Vancampfort et al. 2015a, 2016b), and is more predictive of cardiovascular disease than any other aspect of metabolic health (Myers et al. 2004; $\mathrm{Hu}$ et al. 2005). Health promotion programmes should therefore emphasize the benefit of fitness in order to maximize uptake of exercise in this patient group. Furthermore, interventions should ideally be designed by exercise professionals to ensure that they meet basic principles of exercise prescription, in order to exert significant physiological effects and enable patients to achieve realistic fitness goals.
Patients also valued the psychological effects of exercise, and $75 \%$ of patients viewed stress reduction $/ \mathrm{mood}$ enhancement as motivating factors. Recent meta-analyses have shown that exercise can significantly improve psychological well-being among people with SMI and reduce depression (Rosenbaum et al. 2014; Firth et al. 2015). However, the present study also found that stress, depression and low energy often also act as barriers towards exercise.

The most prominent socio-ecological barrier identified across the studies included in this review was a 'lack of support'. Nonetheless, the majority of patients felt that exercise supervision would enable them to exercise more (Ussher, 2007; Sylvia et al. 2009; Carpiniello et al. 2013). This is congruent with the qualitative literature, within which patients with SMI have stipulated that adequate support can overcome many of the barriers faced towards exercise (Soundy et al. 2014b; Firth et al. 2016b).

Although unsupervised interventions which use less resource-intensive methods (such as education or behavioural change techniques) may seem more cost effective than supervised exercise, this may not be the case for people with SMI. Several recent meta-analyses of exercise interventions in this population have shown that interventions which provide professional support have better adherence to physical activity and significantly greater effects on cardiorespiratory fitness (Vancampfort et al. 2015c, 2016b; Stubbs et al. 2016c). Since both physical activity and fitness are strong predictors of cardiovascular risk and all-cause mortality (Hu et al. 2005; Kodama et al. 2009), supervised interventions which effectively target these variables may ultimately prove more financially worthwhile for improving long-term health outcomes (Vancampfort et al. 2015c, 2016b).

Previous intervention studies have further shown that whereas exercise access and advice is ineffective for increasing physical activity in SMI (Archie et al. 2003; Bartels et al. 2013), providing adequate social support does enable patients to achieve sufficient levels of moderate-to-vigorous exercise (Bartels et al. 2013; Firth et al. 2016c). Although there is currently a lack of cost-effectiveness research examining supervised exercise in SMI, financial reports of exercise interventions for diabetes, mild depression and heart disease indicate that professionally delivered training programmes produce large economic benefits from avoided health system costs (Deloitte Access Economics, 2015).

\section{Limitations}

A strength of these findings is the large number of patients $(n=6431)$ included in the review. Within 
this, there was also substantial ethnic diversity within the included samples, with $50 \%$ belonging to minority groups. However, all of the studies were conducted in western, developed countries, and thus no studies have examined barriers towards exercise among people with SMI in Asia or developing countries. Furthermore, no studies examined differences in motivations or barriers towards exercise between the different ethnic groups within their respective samples. This gap in the literature should be given further consideration in future research, as studies in the general population have shown that beliefs about exercise, and primary reasons for engaging in physical activity, differ significantly between ethnic groups even within the same country (Dergance et al. 2003; ShiuThornton et al. 2004). Specifically, those in minority ethnic groups may face additional challenges towards exercise, such as feeling unsafe in their neighbours (Fahlman et al. 2006) or lacking opportunity to engage in culturally appropriate physical activity (Caperchione et al. 2009). Thus, efforts should be undertaken to identify and provide acceptable physical activity interventions for ethnically diverse populations.

Despite the large total sample, one limitation of this review is that some of the motivations and barriers assessed in meta-analyses were examined by as few as three studies. Additionally, some eligible studies did not provide any proportional data, and thus were not included in the meta-analysis at all. Nonetheless, a full systematic review of each eligible study was also undertaken, for consideration alongside the meta-analytic outputs, in order to provide a complete account of all relevant findings.

It should also be considered that the large majority of patients $(85 \%)$ in this meta-analysis had a diagnosis of schizophrenia, while bipolar disorder and major depressive disorder were relatively under-represented among the eligible studies. Thus, future research should examine if the same motivations and barriers towards exercise identified in this review also generalize to patients with SMIs other than schizophrenia. An online survey study of individuals with high depressive symptoms (but without a confirmed SMI) indicates that our findings will generalize beyond schizophrenia, as the most common barriers towards exercise reported by these individual were again low mood and fatigue (Busch et al. 2015), as was observed in our SMI samples (Fig. 3).

A final limitation is that results are based on selfreported data, derived from questionnaires and surveys administered to patients. Therefore, the results could be affected by response bias, or participants lacking sufficient interest/experience with exercise to accurately describe the barriers faced. The findings from patients' self-report in this study are also congruent with health professionals' opinions, who also acknowledge the importance of social support in overcoming various barriers towards regular exercise (Soundy et al. 2014c).

\section{Conclusion}

People with SMI value exercise for its ability to improve physical health and appearance, and the psychological benefits. However, mental health symptoms, tiredness and insufficient support present substantial barriers for the majority of patients. Taking this into account, exercise training programmes for people with SMI should be designed to improve exercise capacities and cardiorespiratory fitness, while also providing the necessary levels of supervision or assistance for each patient to overcome psychological barriers and achieve their goals. Such interventions would be motivating and rewarding for patients, resulting in higher levels of exercise engagement. This, in turn, could improve physical health outcomes and facilitate functional recovery in SMI.

\section{Supplementary material}

For supplementary material accompanying this paper visit http://dx.doi.org/10.1017/S0033291716001732.

\section{Acknowledgements}

Joseph Firth is funded by an MRC Doctoral Training Scholarship.

\section{Declaration of Interest}

None.

\section{References}

Arango C, Garibaldi G, Marder SR (2013). Pharmacological approaches to treating negative symptoms: a review of clinical trials. Schizophrenia Research 150, 346-352.

Archie S, Wilson JH, Osborne S, Hobbs H, McNiven J (2003). Pilot study: access to fitness facility and exercise levels in olanzapine-treated patients. Canadian Journal of Psychiatry 48, 628-632.

Bartels SJ, Pratt SI, Aschbrenner KA, Barre LK, Jue K, Wolfe RS, Xie H, McHugo G, Santos M, Williams GE (2013). Clinically significant improved fitness and weight loss among overweight persons with serious mental illness. Psychiatric Services 64, 729-736.

Bassilios B, Judd F, Pattison P (2014). Why don't people diagnosed with schizophrenia spectrum disorders (SSDs) get enough exercise? Australasian Psychiatry 22, 71-77.

Busch AM, Ciccolo JT, Puspitasari AJ, Nosrat S, Whitworth JW, Stults-Kolehmainen MA (2015). Preferences for 
exercise as a treatment for depression. Mental Health and Physical Activity 10, 68-72.

Caperchione CM, Kolt GS, Mummery WK (2009). Physical activity in culturally and linguistically diverse migrant groups to Western Society. Sports Medicine 39, 167-177.

Carpiniello B, Primavera D, Pilu A, Vaccargiu N, Pinna F (2013). Physical activity and mental disorders: a casecontrol study on attitudes, preferences and perceived barriers in Italy. Journal of Mental Health 22, 492-500.

Curtis J, Watkins A, Rosenbaum S, Teasdale S, Kalucy M, Samaras K, Ward PB (2016). Evaluating an individualized lifestyle and life skills intervention to prevent antipsychoticinduced weight gain in first-episode psychosis. Early Intervention in Psychiatry 10, 267-276.

Deighton S, Addington J (2014). Exercise practices of young people at their first episode of psychosis. Schizophrenia Research 1, 311-312.

Deloitte Access Economics (2015). Value of Accredited Exercise Physiologists in Australia. Canberra (http://www2. deloitte.com/content/dam/Deloitte/au/Documents/ Economics/deloitte-au-economics-value-exercisephysiologists-Australia.pdf). Accessed 2nd June 2016.

Dergance JM, Calmbach WL, Dhanda R, Miles TP, Hazuda HP, Mouton CP (2003). Barriers to and benefits of leisure time physical activity in the elderly: differences across cultures. Journal of the American Geriatrics Society 51, 863-868.

DerSimonian R, Laird N (1986). Meta-analysis in clinical trials. Controlled Clinical Trials 7, 177-188.

Fahlman MM, Hall HL, Lock R (2006). Ethnic and socioeconomic comparisons of fitness, activity levels, and barriers to exercise in high school females. Journal of School Health 76, 12-17.

Faulkner G, Taylor A, Munro S, Selby P, Gee C (2007). The acceptability of physical activity programming within a smoking cessation service for individuals with severe mental illness. Patient Education and Counseling 66, 123-126.

Firth J, Carney R, Elliott R, French P, Parker S, McIntyre R, McPhee JS, Yung AR (2016c). Exercise as an intervention for first-episode psychosis: a feasibility study. Early Intervention in Psychiatry. Published online: 14 March 2016. doi:10.1111/eip.12329.

Firth J, Carney R, Jerome L, Elliott R, French P, Yung AR (2016b). The effects and determinants of exercise participation in first-episode psychosis: a qualitative study. BMC Psychiatry 16, 36.

Firth J, Cotter J, Elliott R, French P, Yung A (2015). A systematic review and meta-analysis of exercise interventions in schizophrenia patients. Psychological Medicine 45, 1343-1361.

Firth J, Rosenbaum S, Stubbs B, Vancampfort D, Carney R, Yung AR (2016a). Preferences and motivations for exercise in early psychosis. Acta Psychiatria Scandinavica 134, 83-84.

Fraser SJ, Chapman JJ, Brown WJ, Whiteford HA, Burton NW (2015). Physical activity attitudes and preferences among inpatient adults with mental illness. International Journal of Mental Health Nursing 24, 413-420.

Galletly C (2009). Recent advances in treating cognitive impairment in schizophrenia. Psychopharmacology 202, 259-273.
Gardner-Sood P, Lally J, Smith S, Atakan Z, Ismail K, Greenwood K, Keen A, O'Brien C, Onagbesan O, Fung C (2015). Cardiovascular risk factors and metabolic syndrome in people with established psychotic illnesses: baseline data from the IMPaCT randomized controlled trial. Psychological Medicine 45, 1-11.

Gorczynski P, Faulkner G, Greening S, Cohn T (2010). Exploring the construct validity of the transtheoretical model to structure physical activity interventions for individuals with serious mental illness. Psychiatric Rehabilitation Journal 34, 61-64.

Haskell WL, Lee I-M, Pate RR, Powell KE, Blair SN, Franklin BA, Macera CA, Heath GW, Thompson PD, Bauman A (2007). Physical activity and public health: updated recommendation for adults from the American College of Sports Medicine and the American Heart Association. Circulation 116, 1081.

Hoffman BM, Babyak MA, Craighead WE, Sherwood A, Doraiswamy PM, Coons MJ, Blumenthal JA (2011). Exercise and pharmacotherapy in patients with major depression: one-year follow-up of the SMILE study. Psychosomatic Medicine 73, 127-133.

Hu G, Jousilahti P, Barengo NC, Qiao Q, Lakka TA, Tuomilehto J (2005). Physical activity, cardiovascular risk factors, and mortality among Finnish adults with diabetes. Diabetes Care 28, 799-805.

Kane I, Lee H, Sereika S, Brar J (2012). Feasibility of pedometers for adults with schizophrenia: pilot study. Journal of Psychiatric and Mental Health Nursing 19, 8-14.

Kimhy D, Vakhrusheva J, Bartels MN, Armstrong HF, Ballon JS, Khan S, Chang RW, Hansen MC, Ayanruoh L, Lister A (2015). The impact of aerobic exercise on brainderived neurotrophic factor and neurocognition in individuals with schizophrenia: a single-blind, randomized clinical trial. Schizophrenia Bulletin 41, 859-868.

Klingaman EA, Viverito KM, Medoff DR, Hoffmann RM, Goldberg RW (2014). Strategies, barriers, and motivation for weight loss among veterans living with schizophrenia. Psychiatric Rehabilitation Journal 37, 270-276.

Kodama S, Saito K, Tanaka S, Maki M, Yachi Y, Asumi M, Sugawara A, Totsuka K, Shimano H, Ohashi Y (2009). Cardiorespiratory fitness as a quantitative predictor of allcause mortality and cardiovascular events in healthy men and women: a meta-analysis. Journal of the American Medical Association 301, 2024-2035.

Mason OJ, Holt R (2012). Mental health and physical activity interventions: a review of the qualitative literature. Journal of Mental Health 21, 274-284.

McNamee L, Mead G, MacGillivray S, Lawrie SM (2013). Schizophrenia, poor physical health and physical activity: evidence-based interventions are required to reduce major health inequalities. British Journal of Psychiatry 203, 239-241.

Myers J, Kaykha A, George S, Abella J, Zaheer N, Lear S, Yamazaki T, Froelicher V (2004). Fitness versus physical activity patterns in predicting mortality in men. American Journal of Medicine 117, 912-918.

Ribe AR, Laursen TM, Sandbæk A, Charles M, Nordentoft M, Vestergaard M (2014). Long-term mortality of persons with severe mental illness and diabetes: a population-based 
cohort study in Denmark. Psychological Medicine 44, 30973107.

Rosenbaum S, Tiedemann A, Sherrington C, Curtis J, Ward PB (2014). Physical activity interventions for people with mental illness: a systematic review and meta-analysis. Journal of Clinical Psychiatry 75, 964-974.

Scheewe T, Backx F, Takken T, Jörg F, Strater AV, Kroes A, Kahn R, Cahn W (2013). Exercise therapy improves mental and physical health in schizophrenia: a randomised controlled trial. Acta Psychiatrica Scandinavica 127, 464-473.

Sherwood NE, Jeffery RW (2000). The behavioral determinants of exercise: implications for physical activity interventions. Annual Review of Nutrition 20, 21-44.

Shiu-Thornton S, Schwartz S, Taylor M, LoGerfo J (2004). Older adult perspectives on physical activity and exercise: voices from multiple cultures. Preventing Chronic Disease 1, A09.

Soundy A, Freeman P, Stubbs B, Probst M, Coffee P, Vancampfort D (2014a). The transcending benefits of physical activity for individuals with schizophrenia: a systematic review and meta-ethnography. Psychiatry Research 220, 11-19.

Soundy A, Freeman P, Stubbs B, Probst M, Vancampfort D (2014b). The value of social support to encourage people with schizophrenia to engage in physical activity: an international insight from specialist mental health physiotherapists. Journal of Mental Health 23, 256-260.

Soundy A, Stubbs B, Probst M, Hemmings L, Vancampfort D (2014c). Barriers to and facilitators of physical activity among persons with schizophrenia: a survey of physical therapists. Psychiatric Services 65, 693-696.

StatsDirect L (2005). StatsDirect Statistical Software. StatsDirect: UK.

Stubbs B, Firth J, Berry A, Schuch FB, Rosenbaum S, Gaughran F, Veronesse N, Williams J, Craig T, Yung AR, Vancampfort D (2016a). How much physical activity do people with schizophrenia engage in? A systematic review, comparative meta-analysis and meta-regression.

Schizophrenia Research. Published online: 31 May 2016. doi:10.1016/j.schres.2016.05.017.

Stubbs B, Vancampfort D, Rosenbaum S, Ward PB, Richards J, Soundy A, Veronese N, Solmi M, Schuch FB (2016c). Dropout from exercise randomized controlled trials among people with depression: a meta-analysis and meta regression. Journal of Affective Disorders 190, 457-466.

Stubbs B, Williams J, Gaughran F, Craig T (2016b). How sedentary are people with psychosis? A systematic review and meta-analysis. Schizophrenia Research 171, 103-109.

Suetani S, Waterreus A, Morgan V, Foley D, Galletly C, Badcock J, Watts G, McKinnon A, Castle D, Saha S (2016). Correlates of physical activity in people living with psychotic illness. Acta Psychiatrica Scandinavica.

Sylvia LG, Kopeski LM, Mulrooney C, Reid J, Jacob K, Neuhaus EC (2009). Does exercise impact mood? Exercise patterns of patients in a psychiatric partial hospital program. Journal of Psychiatric Practice 15, 70-78.

Ussher M (2007). Physical activity preferences and perceived barriers to activity among persons with severe mental illness in the United Kingdom. Psychiatric Services 58, 405-408.

Vancampfort D, Correll CU, Probst M, Sienaert P, Wyckaert S, De Herdt A, Knapen J, De Wachter D, De Hert M (2013b). A review of physical activity correlates in patients with bipolar disorder. Journal of Affective Disorders 145, 285-291.

Vancampfort D, De Hert M, Sweers K, De Herdt A, Detraux J, Probst M (2013a). Diabetes, physical activity participation and exercise capacity in patients with schizophrenia. Psychiatry and Clinical Neurosciences 67, 451-456.

Vancampfort D, Firth J, Schuch F, Rosenbaum S, De Hert M, Mugisha J, Probst M, Stubbs B (2016a). Physical activity and sedentary behavior in people with bipolar disorder: a systematic review and meta-analysis. Journal of Affective Disorders 201, 145-152.

Vancampfort D, Knapen J, Probst M, Scheewe T, Remans S, De Hert M (2012). A systematic review of correlates of physical activity in patients with schizophrenia. Acta Psychiatrica Scandinavica 125, 352-362.

Vancampfort D, Rosenbaum S, Probst M, Soundy A, Mitchell A, De Hert M, Stubbs B (2015a). Promotion of cardiorespiratory fitness in schizophrenia: a clinical overview and meta-analysis. Acta Psychiatrica Scandinavica 132, 131-143.

Vancampfort D, Rosenbaum S, Schuch FB, Ward PB, Probst M, Stubbs B (2015c). Prevalence and predictors of treatment dropout from physical activity interventions in schizophrenia: a meta-analysis. General Hospital Psychiatry. Published online: 2 December 2015. doi:10.1016/j. genhosppsych.2015.11.008.

Vancampfort D, Rosenbaum S, Schuch FB, Ward PB, Richards J, Mugisha J, Probst M, Stubbs B (2016b). Cardiorespiratory fitness in severe mental illness: a systematic review and meta-analysis. Sports Medicine (in press).

Vancampfort D, Stubbs B, Mitchell AJ, De Hert M, Wampers M, Ward PB, Rosenbaum S, Correll CU (2015b). Risk of metabolic syndrome and its components in people with schizophrenia and related psychotic disorders, bipolar disorder and major depressive disorder: a systematic review and meta-analysis. World Psychiatry 14, 339-347.

Ward M, White D, Druss B (2015). A meta-review of lifestyle interventions for cardiovascular risk factors in the general medical population: lessons for individuals with serious mental illness. Journal of Clinical Psychiatry 76, 477-486.

Wynaden D, Barr L, Omari O, Fulton A (2012). Evaluation of service users' experiences of participating in an exercise programme at the Western Australian State Forensic Mental Health Services. International Journal of Mental Health Nursing 21, 229-235. 